\title{
Erratum to: A phase II open-label randomized study to assess the efficacy and safety of selumetinib (AZD6244 [ARRY-142886]) versus capecitabine in patients with advanced or metastatic pancreatic cancer who have failed first-line gemcitabine therapy
}

\author{
György Bodoky • Constanta Timcheva • \\ David Robert Spigel • Phillip Joseph La Stella • \\ Tudor Eliade Ciuleanu • G. Pover • N. C. Tebbutt
}

Published online: 26 July 2011

(C) Springer Science+Business Media, LLC 2011

Erratum to: Invest New Drugs

DOI 10.1007/s10637-011-9687-4

An error has occurred during production of this article. In Table 1 the published version states: selumetinib, $n=32$; capecitabine, $n=38$. This should be: selumetinib, $n=38$; capecitabine, $n=32$. The correct Table 1 is also shown on the next page.

The online version of the original article can be found at http://dx.doi. org/10.1007/s10637-011-9687-4.

G. Bodoky $(\bowtie)$

Department of Oncology, St. László Hospital,

Gyáli út 5-7,

1097 Budapest, Hungary

e-mail: bodokygy@hungarnet.hu

C. Timcheva

National Oncology Centre, Clinic of Chemotherapy,

5 Plovdivsko pole St.,

1756 Sofia, Bulgaria

\section{R. Spigel}

Sarah Cannon Research Institute, (SCRI),

250 25th Avenue North, Suite 110,

Nashville, TN 37203, USA

\section{P. J. La Stella}

St. Joseph Mercy Hospital Cancer Care Center,

5301 East Huron River Drive, P.O. Box 995,

Ann Arbor, MI 48106-0995, USA
T. E. Ciuleanu

Day Hospital Department-Medical Oncology,

Oncological Institute I Chiricuta,

Republicii 34-36 str,

Cluj-Napoca 40015, Romania

G. Pover

AstraZeneca,

Alderley Park, UK

N. C. Tebbutt

Austin Heath,

Heidelberg, VIC 3084, Australia 
Table 1 Patient demographics and baseline characteristics (intent-to-treat population)
Characteristic

Number of patients (\%)

Selumetinib $n=38$

Capecitabine $n=32$

Age (years)

Mean (standard deviation)

Median (range)

Sex

Male

Female

Race

Caucasian

Oriental

Previous treatments

Radiation

Surgery

Gemcitabine

Number of metastatic sites

0

1

$>1$
$63.1(8.13)$

$65(48-80)$

24 (63)

$14(37)$

$36(95)$

2 (5)

$5(13)$

$29(76)$

38 (100)

$3(8)$

$13(34)$

$22(58)$
6 (19)

25 (78)

32 (100)

2 (6)

14 (44)

$62.0(11.85)$

$62(20-79)$

11 (34)

21 (66)

$32(100)$

0

$16(50)$ 